\title{
Evolución del desarrollo psicomotor en niños menores de 4 años en situación de abandono
}

\author{
Marica Bussi ${ }^{1}$
}

Bussi M. Evolución del desarrollo psicomotor en niños menores de 4 años en situación de abandono. Cuid salud, ene-jun 2014; 1(1).

\section{RESUMEN}

Objetivo: describir la evolución del desarrollo psicomotor de niños menores de 4 años en situación de abandono, en aparente retraso residentes en un albergue religioso. Metodología: enfoque cuantitativo, método observacional, diseño exploratorio descriptivo, de corte prospectivo; realizado en la Casa Hogar de Villa el Salvador que brinda atención integral a niños con apoyo de un equipo multiprofesional que estimula el desarrollo psicomotor, afectivo y espiritual; la población lo constituyeron 20 niños albergados en la Casa Hogar. Los datos fueron recolectados entre julio-diciembre 2011 utilizando como instrumento una ficha de registro para los datos sociodemográficos, observación participante y dos escalas de evaluación del desarrollo psicomotor (EEDP y TEPSI). El análisis de datos fue mediante la estadística descriptiva. Resultados: el desarrollo psicomotor de los niños mejoró, siendo que en los datos basales el $40 \%$ de niños fue calificado como normal y $25 \%$ en retraso. Al finalizar el estudio, un $83,3 \%$ de niños alcanzó la calificación de normalidad y solo el 16,7\% estaba en riesgo. Según áreas, hubo mejoría en la motora $(p=00,49)$ y lenguaje $(p=0,0016)$. Conclusiones: el desarrollo psicomotor de los niños mejoró ostensiblemente, destacando el trabajo colaborativo del equipo multiprofesional, con impacto en el desarrollo integral del menor.

Palabras clave: retraso psicomotor, niños en abandono, maltrato infantil, enfermería.
Bussi M. Evolution of psychomotor development in children under 4 years old in neglect situation. Cuid salud, ene-jun 2014; 1(1).

\begin{abstract}
Objective: To describe the evolution of the psychomotor development of children under 4 years old in neglect situation with in apparent retardation in a religious shelter. Methodology: quantitative focus, observational method, prospective and descriptive exploratory design; the study has been done at "Casa Hogar de Villa el Salvador" that provides integral care to children with the support of a multidisciplinary team that stimulates the psychomotor, affective and spiritual development; the population was constituted by 20 children from the home. Data was collected from July to December 2011, using a registration card for socio-demographic data, participant observation and two scales of psychomotor development evaluation (EEDP and TEPSI). Data analysis was by descriptive statistics. Results: the psychomotor development of children improved, at the beginning the $40 \%$ of children were classified as normal and $25 \%$ in retardation. At the end of the study, $83.3 \%$ of children reached the calcification of normality and only $16.7 \%$ was at risk. According areas, there was an improvement in motor area $(\mathrm{p}=00.49)$ and language $(\mathrm{p}$ $=0.0016$ ). Conclusions: the psychomotor development of children improved significantly, highlighting the collaborative work of the multidisciplinary team, with an impact on the integral development of the child.
\end{abstract}

Keywords: psychomotor development, abandoned children, child abuse, nursing.

\footnotetext{
1 Licenciada Enfermera. Egresada de la Escuela de Enfermería Padre Luis Tezza, afiliada a la Universidad Ricardo Palma, LimaPerú. Religiosa de la Congregación Italiana Hermanas Misioneras de la Resurrección. Casa Hogar Villa El Salvador.
} 


\section{INTRODUCCIÓN}

Actualmente, vivimos sumergidos en una sociedad llena de violencia, problemática mundial, al cual desafortunadamente no escapan los niños. El abandono es una forma de maltrato infantil y constituye un problema universal alarmante y en continuo aumento. Se trata de una situación de negligencia infantil, en la cual el grado es extremo y cuyas consecuencias físicas en el niño son elevadas. ${ }^{1}$

En los Estados Unidos, ${ }^{2}$ a finales de los 90 se estimó que había 900000 niños maltratados, con una incidencia de 12,9 por cada 1000 niños, siendo que 12000 mueren anualmente por alguna forma de maltrato, destacándose que el 53,7\% había sido abandonado, $22 \%$ recibió maltrato físico y $11,5 \%$ abuso sexual; con una incidencia mayor de casos entre los más pequeños ( $78 \% \leq 3$ años de edad).

En el Perú, en el 2009, el Instituto Nacional de Salud $^{3}$ reportó que había registrado cerca de $40 \%$ casos de maltrato infantil, físico y psicológico causados por las madres en el hogar. La representante del Instituto comentó que solo en el primer semestre de ese año ya habían recibido 200 casos de violencia infantil, de los cuales $31 \%$ era por negligencia o abandono, $11 \%$ por maltrato físico y $12 \%$ por maltrato psicológico.

La población de niños y niñas, jóvenes y adolescentes en el país alcanza a 10 millones 67 mil personas, representando el $30,5 \%$ de la población según estadística del INEI $2010 .^{4}$ Reconociéndose que la niñez es el grupo más vulnerable para vivir situaciones de riesgo, abandono y condición de pobreza y que el $10 \%$ de la población de cualquier país se constituye de personas con algún riesgo de discapacidad permanente, física o mental; limitando una o más actividades de su vida diaria. ${ }^{5}$ Actualmente, se desconoce cuántos niños tienen retraso en el desarrollo mental, motor, social y emocional con deficiente estado de salud y nutrición.

Según la ENDES 2011, ${ }^{6}$ el 17,9\% de $\leq 5$ años presenta desnutrición crónica y 50,3\% de los niños entre 6-36 meses anemia nutricional, quedando implícito que los mismos tendrán serias deficiencias en su desarrollo y crecimiento; no obstante, se desconoce la magnitud del problema.

La Convención sobre los Derechos del Niño refiere que se debe proteger al niño contra toda forma de perjuicio o abuso físico o mental, descuido o trato negligente, malos tratos o explotación, mientras el niño se encuentra bajo la custodia de sus padres o tutora. ${ }^{7}$ Siendo que el abandono como es una forma de maltrato o perjuicio para el niño constituido por abandono material o físico, emocional y moral. El primero se refiere a un maltrato infantil pasivo, en el que las necesidades físicas básicas de alimentación, vestido, protección y vigilancia, en situaciones peligrosas o en relación a los cuidados del menor, no son atendidas temporal o permanentemente. En el segundo, calificado como pasivo, el niño deja de recibir afecto, apoyo, y todo lo necesario para crecer y desarrollarse psicológicamente sano, estando bloqueado el contacto afectivo del niño, lo que repercute negativamente en su formación humana. $^{2,8}$

Existen diversas formas de abandono, destacándose en el estudio el social, económico, emocional y familiar. En el abandono social se considera la prostitución, drogadicción, alcoholismo, inestabilidad emocional, falta de planificación familiar y conductas irresponsables que llevan a embarazos indeseados $y$ por consiguiente, al abandono. En el económico, se incluyen las familias pobres, quienes no tienen acceso a servicios básicos y, en general, viven en situación de desempleo. En el abandono emocional, está la incapacidad de los padres de enfrentar los problemas por inmadurez emocional, baja autoestima, falta de experiencia e inseguridad. En el familiar, existen problemas de desintegración y separación de los padres por falta de comunicación, destacando el caso de las madres adolescentes que abandonan a sus hijos por falta de apoyo material. ${ }^{8} \mathrm{El}$ abandono emocional, a corto y largo plazo, puede ser más debilitante que el físico; el abandono afectivo, especialmente de la madre, tiene impacto en el desarrollo y crecimiento normal del niño, provocando retraso psicomotor $\mathrm{y}$ deterioro de sus facultades mentales, principalmente del área cognitiva. ${ }^{9}$

Se define retraso psicomotor (RPM) a la lentitud y/o anormalidad de las adquisiciones de los primeros hitos del desarrollo (primeros 36 meses de vida), que deviene de un agresión al sistema nervioso central (SNC), o determinado genéticamente, en general por parálisis cerebral, retraso mental, trastornos específicos del desarrollo, pobre estimulación ambiental, enfermedades crónicas, entre otras. ${ }^{10}$

Algunos estudios muestran el impacto negativo del abandono. Entre las familias pobres 
existe carencia de estimulación, siendo que el $10 \%$ de los niños había sufrido de maltrato emocional antes del abandono físico; ${ }^{1}$ quedando demostrado que el maltrato afecta el desarrollo evolutivo normal del niño, con problemas en la conducta y en las relaciones sociales. ${ }^{11}$

Frente a esta realidad y la repercusión que tiene el abandono físico y emocional en los niños, la Casa Hogar de Villa El Salvador, institución que alberga niños menores de 4 años en situación de abandono, brinda un cuidado integral desde la asistencia básica hasta el desarrollo integral psicomotor y cognitivo, contando con un equipo multiprofesional conformado por una terapista física, un pediatra, un psicólogo, una asistente social, enfermeras y misioneras. En ese sentido, este estudio tuvo como objetivo describir la evolución del desarrollo psicomotor de los niños en situación de abandono, albergados en la Casa Hogar de Villa El Salvador.

\section{METODOLOGÍA}

Estudio de enfoque cuantitativo, método observacional, diseño exploratorio descriptivo, de corte prospectivo; realizado en la Casa Hogar Villa El Salvador, localizado en el sector 9 de la Encantada, distrito del Villa El Salvador-Lima. Dicha institución es parte de una comunidad religiosa, habitada por jóvenes misioneras y consagradas, quienes se responsabilizan de la vida y educación integral (cuerpo, mente y espíritu) de niños en situación de abandono que llegan a la Casa Hogar, los cuales además son asistidos por el equipo multiprofesional, recibiendo estimulación psicomotriz continua, acorde con la edad del niño y sus necesidades.

La mayoría de niños de la Casa Hogar fue encontrada por la policía, entidad que inicialmente los conduce a la Unidad de Investigación Tutelar, entidad encargada de buscar instituciones de acogida temporal. Dichos niños pueden volver con sus familiares en caso de ser localizados y si éstos así lo desean; de lo contrario son declarados en abandono por el Ministerio de la Mujer y Desarrollo Social (MIMDES) y quedan a la espera de proceso de adopción a través la Secretaria Nacional de Adopciones de Lima.

La población fue constituida por todos los niños $\leq 4$ años de edad albergados en la Casa Hogar ( $\mathrm{N}=20)$, quienes tenían características en común y estaban en aparente retraso psicomotor; habiéndose excluido a los niños menores de 6 meses de edad, niños con enfermedad discapacitante y con $\leq 6$ meses de estancia en la Casa.

En la recolección de datos se utilizó como técnica la observación participante y como instrumento, una guía de observación estructurada en cuatro áreas (motora fina, motora gruesa, lenguaje y social), la cual fue aplicada a los niños mensualmente durante el periodo de estudio, observándose las características y condiciones de cada niño, además de comportamientos, actitudes, habilidades, comunicación (verbal/no verbal) y ambientales. Se contó también, con una ficha de registro para recolectar información sobre los antecedentes de maltrato o condición de ingreso del menor a la Casa Hogar, además de recopilar datos actuales correspondientes a su desarrollo integral, de salud y otros significativos.

Para evaluar el desarrollo psicomotor se utilizaron las escalas de evaluación del Ministerio de Salud (MINSA). Para el $\leq 2$ años de edad se consideró la EEDP (Escala de Evaluación del Desarrollo Psicomotor), que contiene 75 ítems asignándose a cada ítem 6 puntos en el $<1$ año y, a partir del año, cada ítem equivale a 12 puntos; a los 15 meses el valor alcanza a 18 puntos por ítem. En este instrumento, previamente se calcula la edad cronológica (EC) y mental (EM) del niño, dividiéndose estas medidas para obtener la razón (R), la cual se convierte en un puntaje estándar utilizando tablas preestablecidas para clasificar el desarrollo del menor: $\leq 85$ puntos (niño normal), entre 85-90 puntos (niño en riesgo), $\leq 69$ puntos (niño con retraso).

En los niños de 2-5 años se utilizó la escala de TEPSI (Test de Evaluación Psicomotora y Social), compuesta de 52 ítems, organizados en tres sub-escalas: coordinación (16 ítems), lenguaje (24 ítems) y motricidad (12 ítems). Los resultados se clasificaron en: de 20-30 puntos niños con retraso, entre $30-40$ puntos niños en riesgo y entre 40-80 puntos niños normales.

La Ficha de registro y la Guía de observación fueron sometidas a juicio de expertos, entre enfermeras, pediatras, terapista físico y psicólogo, quienes sugirieron realizar algunas mejoras. La prueba binomial de esta evaluación mostró resultados significativos en la mayoría de los ítems.

Obtenida la autorización de la directora de la Casa Hogar, se procedió a recolectar los datos 
entre junio-diciembre del 2011, en un ambiente previamente preparado dentro de la Casa Hogar, destacando que el área cuenta con suficiente material para motivar el desarrollo psicomotor de los niños. Previamente se recolectó información de los expedientes de cada niño, identificando datos sociales, psicológicos y médicos; luego se realizó la evaluación de los niños según grupo etario. Para la evaluación psicomotora se solicitó la participación de una tercera persona, ajena al estudio, función que desempeño en todas las evaluaciones realizadas a los niños.

Para el análisis de la información, los datos se codificaron en el programa Excel 2007, de forma dupla para el control de calidad. Para procesarlos se usó el software estadístico SPSS (Statistical Packagefor The Social Sciences) versión 5.0 mediante test estadísticos descriptivos (frecuencias, porcentajes, promedios, desviación estándar), además de pruebas para verificar asociación y tendencias de mejora en las evaluaciones a través la prueba de Chi cuadrado y de Pearson. Destacándose que el proyecto fue aprobado por el Comité de investigación y ética del Instituto Nacional de Salud del Niño (INSN), cuyo documento de respuesta fue Carta $N^{\circ} 1062$ OEAIDE-INSN-2011.

\section{RESULTADOS}

En los datos sociodemográficos, se resalta que el $60 \%$ era varón y $45 \%$ (9) $\leq 1$ año, siendo 2 mujeres y 7 varones, el $25 \%$ (5) tenía entre $1-2$ años y otros entre 2-3 años, solo 1 (5\%) era >3 años. Un 95\% (19) de los niños había ingresado a la Casa Hogar por situación de abandono y uno por violencia; una niña procedía de familia desintegrada y fue abandonada por su madre después de haberle intentado envenenar.

En la evaluación psicológica, al ingreso a la Casa Hogar, un 40\% (8) de niños era sociable (entre estos 6 eran < 1 año y dos de 1-2 años), 35\% era temeroso, $20 \%$ (4) hiperactivo y $5 \%$ (1) violento. Según el análisis psicológico evolutivo al iniciar el estudio, un 55\% (11) se relacionaba bien (entre éstos, 5 fueron < 1 año), 25\% (5) tenía rasgos de desconfianza, $15 \%$ (3) rasgos de seguridad y $5 \%$ (1) se aislaba.

En el control antropométrico, al ingreso a la Casa Hogar, un70\% (14) era normal, 10\%(2) tenía bajo peso y $20 \%$ (4) estaba desnutrido. Datos que habían variado al iniciar el estudio, ya que el $95 \%$ (19) estaba en condición de normalidad y solo
5\%(1) tenía bajo peso. Durante el estudio fueron perdidos dos niños (10\%), quienes habían sido recogidos por sus mamas; a seguir se describe los resultados del estudio.

Tabla 1: Comparación del desarrollo psicomotor según grupo etario en los niños de la Casa Hogar Villa El Salvador. Diciembre 2011.

\begin{tabular}{ccccccc}
\hline \multicolumn{3}{c}{ EVALUACIÓN DEL DESARROLLO PSICOMOTOR } \\
$\begin{array}{c}\text { Grupos } \\
\text { etarios }\end{array}$ & $\begin{array}{c}\text { Normal } \\
\text { Basal }\end{array}$ & Final & \multicolumn{2}{c}{ Riesgo } & \multicolumn{2}{c}{ Retraso } \\
\hline$<$ 1 año & 3 & 6 & 5 & 1 & 1 & - \\
$1-2$ años & 1 & 3 & - & 2 & 4 & - \\
2 - 3 años & 4 & 5 & 1 & - & - & - \\
$>3$ años & - & 1 & 1 & - & - & - \\
Total & 8 & 15 & 7 & 3 & 5 & - \\
Porcentaje & 40 & 83,3 & 35 & 16,7 & 25 & - \\
$P$ & $0,7817^{*}$ & $0,1101^{* *}$ & & \\
\hline *Prueba de chi Cuadrado, **Prueba de Pearson &
\end{tabular}

En la tabla 1 se observa los resultados del coeficiente del desarrollo psicomotor alcanzado por los niños según grupo etario y estadio a lo largo del estudio: normal, riesgo y retraso, evidenciándose que hubo mejoría global en todos los grupos, aunque no se observan diferencias significativas en el transcurso de los seis meses (normal $p=0,7817$ y riesgo $p=0,1101$ ).

Al realizar el análisis por áreas de desarrollo psicomotor, se observa en el gráfico 1 que en julio el 49,8\% (9) de niños tenía una condición de desarrollo normal y $25 \%$ (5) fue calificado en retraso. No obstante, al concluir el estudio el $61,1 \%$ (11) de los niños llegó al estado de normalidad, demostrando cambios significativos al compararse con los datos basales $(p=0,0016)$.

Gráfico 1: Comparación del desarrollo psicomotor en el área de lenguaje en los niños de la Casa Hogar Villa El Salvador. Diciembre 2011.

$$
p=0,0016
$$

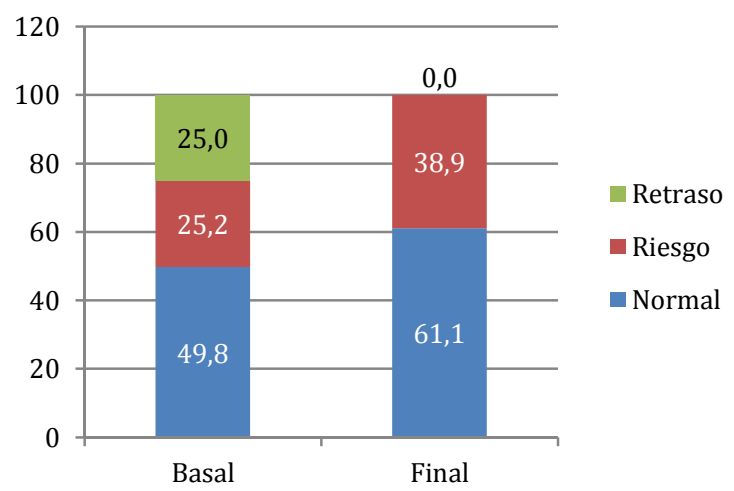


no evalúa de forma independiente esta área. Los datos del gráfico 2 muestran avances entre los datos basales y finales en el desarrollo psicomotor de los niños, pero sin diferencias significativas ( $p=$ 0,111 ). De ese modo, en julio el $44 \%$ (5) tenía una condición de normalidad y $33 \%$ (4) tenía retraso, incrementándose la proporción de niños normales al finalizar el estudio a 77,8\% (7), desapareciendo la condición de niño con retraso. Se destaca que tres niños $\leq 2$ años pasaron a ser evaluados con el TEPSI al final del estudio por edad cronológica.

Gráfico 2: Comparación del desarrollo psicomotor en el área social en los niños de la Casa Hogar Villa El Salvador. Diciembre 2011.

$$
p=0,111
$$

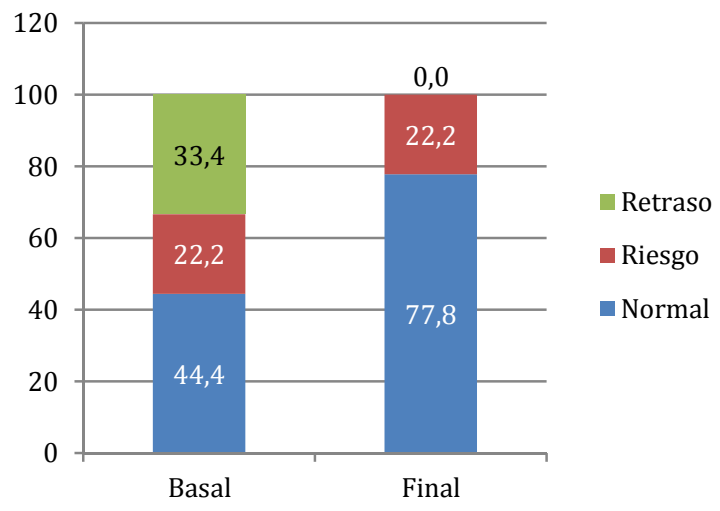

Gráfico 3: Comparación del desarrollo psicomotor en el área motora en los niños de la Casa Hogar Villa El Salvador. Diciembre 2011.

$$
p=0,049
$$

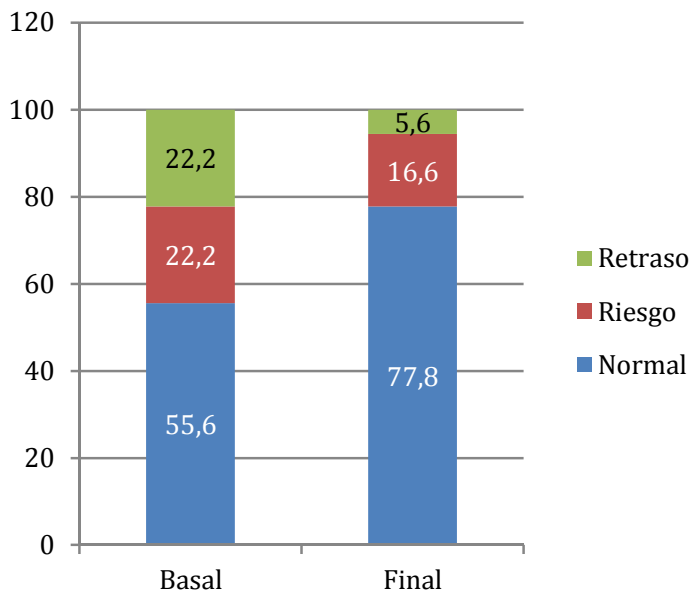

Ya en el gráfico 3, se observa cambios significativos en el desarrollo psicomotor de los niños al finalizar el estudio $(p=0,049)$. Los datos muestran que en julio el 55,6\% (12) de los niños estaba en condición de normalidad y un $22,2 \%$ (4) en retraso, siendo que al mes de diciembre el $77,8 \%$ (14) habia alcanzado una condición psicomotora normal (18 niños).

Gráfico 4: Comparación del desarrollo psicomotor en el área de coordinación en los niños de la Casa Hogar Villa El Salvador. Diciembre 2011.

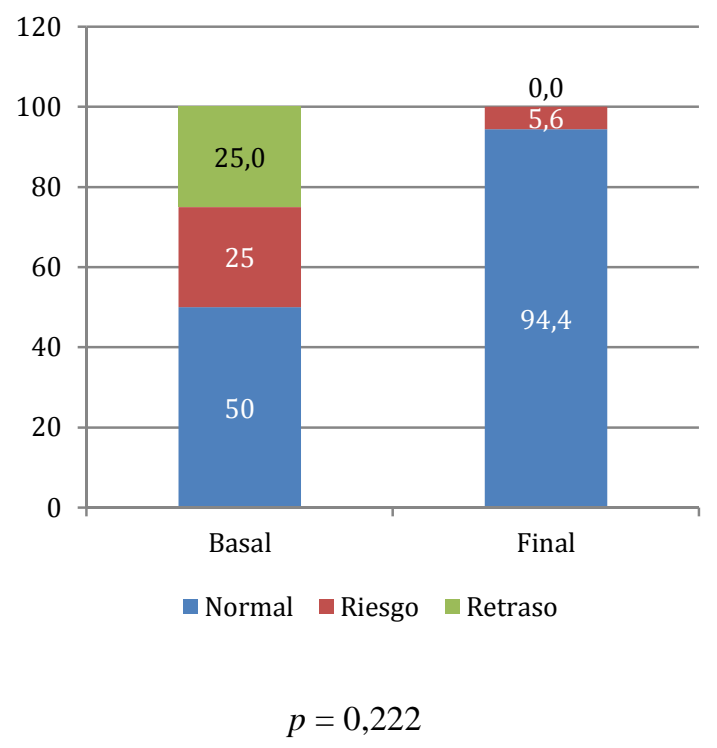

En el gráfico 4, en julio el $50 \%$ (10) de los niños estaba en condición de normalidad en su desarrollo psicomotor y $25 \%$ (5) de niños estaba con retraso. Al finalizar el estudio, el 94,4\% (17) llegó a una condición de normalidad y el 5,6\% (1) estaba en riesgo, aunque a pesar del cambio en la mayoría de los niños no se observan diferencias significativas entre los datos bases y finales $(p=$ 0,222).

Finalmente, en la tabla 2 se observa la evaluacion del desarrollo psicomotor por escalas comparando los datos basales y finales, identificandose que en ambos grupos etarios de niños hubo cambios significativos a lo largo del estudio. Según escala, con el TEPSI en el mes de julio los niños tenían una edad media de $41 \mathrm{~m} \pm 9 \mathrm{y}$ en diciembre tuvieron $52 \mathrm{~m} \pm 9$. En la escala de EEDP, en el mes de julio la edad media fue de 78 $\mathrm{m} \pm 16$ y en diciembre llegó a $90 \pm 11 \mathrm{~m}$. 
Tabla 2: Comparación del desarrollo psicomotor según instrumento en los niños de la Casa Hogar Villa El Salvador. Diciembre 2011.

\begin{tabular}{cccccc}
\hline & Basal Julio & Final Diciembre & ${ }^{*} \mathbf{p}$ \\
$\begin{array}{c}\text { Escala de } \\
\text { Evaluación }\end{array}$ & $\mathbf{n}$ & $\overline{\mathbf{X}} \pm \mathbf{D E}$ & $\mathbf{n}$ & $\overline{\mathbf{X}} \pm \mathbf{D E}$ & Significativo \\
& & & & & \\
\hline EEDP & 14 & $78 \pm 16$ & 9 & $90 \pm 11$ & 0,032 \\
TEPSI & 6 & $41 \pm 9$ & 9 & $52 \pm 9$ & 0,019 \\
Total & 20 & & 18 & & \\
\hline $\mathrm{p} \leq 0,05$ & & & & &
\end{tabular}

\section{DISCUSIÓN}

Martínez ${ }^{8}$ refiere que los niños al nacer tienen millones de células cerebrales que se multiplican rápidamente cuando el neonato entra en contacto con la estimulación externa y son reconocidas como base para el aprendizaje. La falta de estimulación, además de impedir la proliferación de células, puede ocasionar efectos irreversibles en el desarrollo del cerebro, entre ellos, la desnutrición en el primer año de vida conlleva a un inadecuado crecimiento y desarrollo y mayor propensión a las enfermedades.

Sobre el desarrollo cerebral De Bea, ${ }^{12}$ destaca la plasticidad cerebral del niño al nacer, lo que permite al cerebro crecer según las interacciones externas que recibe y las experiencias que vive el niño; estos procesos construyen la estructura del cerebro desarrollando sus funciones mentales y psíquicas. El desarrollo de las neuronas, sus dendritas y la mielinización dependen, desde el principio de la vida, de la cantidad y calidad de estímulos que el niño recibe; siendo probable que en los niños institucionalizados se produzca un menor desarrollo de la arborización dendrítica, causando pérdida de las conexiones y empobrecimiento de las capacidades psíquicas del niño en las áreas social, emocional, intelectual y de aprendizaje.

En el caso de los niños de la Casa Hogar, inicialmente éstos se muestran como apagados y llorones, vociferando y gritando por encontrarse en un lugar desconocido. Ya durante su permanencia comienzan a manifestar confianza, ayudando mucho en esto las actividades de socialización que se desarrollan en conjunto con todos ellos, recibiendo estímulos motrices y psicosociales por parte de personal enfermero y de las hermanas consagradas y misioneras.
En el estudio, casi todos los niños provenían de situaciones de abandono (95\%) y fueron encontrados en las calles, veredas, puentes y en cajas de cartón en los parques de la ciudad; otras veces, los niños habían sido retirados de padres alcohólicos y/o familias disfuncionales, reflejando la grave crisis que pueden pasar muchas familias en el Perú.

Al respecto, el Obispo de Arequipa, Javier del Rio, ${ }^{13}$ afirma que la "crisis de la familia es fruto de la crisis del hombre", recordando que la familia fue creada por Dios y es fruto del amor, fundado en la verdad; la crisis proviene de una mentalidad de la sociedad consumista que busca desordenadamente el confort y el placer, de una visión egoísta e individualista de la persona que solo piensa en sí misma. A su vez, Juan Pablo II decía que la familia es la célula primera y vital de la sociedad, cuya misión es custodiar, revelar y comunicar amor, sin el cual ningún ser humano puede vivir; es la fuerza necesaria para la comunión, la unidad que se manifiesta en la fidelidad, respeto, perdón, don gratuito de la vida para el otro.

Madre Elvira, ${ }^{14}$ fundadora de la Comunidad Cenáculo, nombre original de la Asociación Religiosa de la Casa Hogar, respecto a la familia decía "la familia es una perla preciosa, es la riqueza de todo ser humano, en un tiempo lleno de agitación y confusión, miedos y rebeliones. En los albores de la creación, cuando Dios creó los cielos, los mares, los montes y toda la belleza que os circunda, su amor se complacía en preparar el hábitat natural para dar vida al hombre y a la mujer"; afirmaba que aunque los niños hayan sido abandonados, de todas maneras sus madres les han dado la vida, que es el don más precioso que cada ser humano tiene, por lo tanto, tienen el derecho de encontrar a Dios, el derecho a conocer la belleza de la vida, de que el amor y la paz existen, por eso el propósito principal de la Casa Hogar es cuidar y educar a los niños en la fe y valores cristianos, brindándole el amor de una familia.

En los datos basales, el 55,6\% de los niños se relacionaba bien, $5 \%$ manifestaba seguridad y otro $25 \%$ mantenía una actitud de desconfianza; siendo posible que dicha actitud sea consecuencia del abandono experimentado, con falta de cariño y afecto por parte de los padres, además del maltrato físico y emocional traducidos en insultos, terror y amenazas. Era muy común en los niños los gestos agresivos como morder, pegar, empujar, gritar, entre otros. 
Según Erikson, citado por Gadea, ${ }^{15}$ en la primera etapa del ciclo vital se debe desarrollar la confianza básica; los niños al ser alimentados y cuidados amorosamente desarrollan un sentimiento de bondad interior que depende de la calidad de la relación que tienen con sus padres o cuidadores; además, la confianza es un requisito para que los niños adquieran su autonomía en el transcurso de la vida.

El método Montessori, ${ }^{10}$ creado en Italia en 1907 por la primer médico mujer y actualmente muy utilizado en los colegios, destaca que la confianza el niño la adquiere a través de las leyes de la vida; debiendo ser educados con libertad en un entorno preparado para alcanzar su desarrollo integral, actuando con libertad, inteligencia y dignidad, dejándole escoger en el medio ambiente material para su actividad; libertad que le ayuda a tomar decisiones. Los niños poseen especial sensibilidad para observar y adaptarse a diversos medios, especialmente en ambientes rodeados de impresiones sanas y positivas, experimentando un cambio extraordinario en sus comportamientos, por esta característica la autora del método llamó a los niños "mentes brillantes". En la Casa Hogar los niños experimentan ese cambio con el tiempo, favorecido por la dedicación, el amor, el respeto, la vida familiar y la oración que les ofrecen las hermanas consagradas y misioneras.

Esta relación de familia, muchas veces crea un gran apego de los niños con las misioneras, siendo difícil para los niños la vivencia de una separación. Bolwby y Aisworth, en la teoría del apego, citado por Oliva, ${ }^{16}$ plantean que la separación entre un niño pequeño y una figura de apego es perturbadora, provocando en ellos miedos muy intensos y un cierto grado de ansiedad. Hecho observado en la mayoría de niños de la Casa Hogar, sin embargo, los niños tienen mucha capacidad de sufrir y adaptarse al cambio, viven el momento presente, son más capaces de perdonar y saben recomenzar fácilmente.

Respecto a los datos antropométricos, durante el estudio los niños padecieron de un episodio de rotavirus, debiendo recibir tratamiento, que tuvo impacto en el estado nutricional, sobre todo en los más pequeños, afectando la evolución del desarrollo psicomotor. De ese modo, al finalizar el estudio el $94,4 \%$ de niños tenía un desarrollo antropométrico normal y solo un 5,6\% tenía bajo peso. Resaltando que la mayoría de niños no había recibido lactancia materna.
En el desarrollo psicomotor (DPM), los niños mostraron mejoría (tabla 2), pues al inicio el $40 \%$ (8) era normal y $25 \%$ (5) tenía retraso; datos que resaltan la evolución favorable gracias al trabajo del equipo multiprofesional en acción colaborativa entre terapista físico, psicólogo, enfermeras $\mathrm{y}$ misioneras.

En el área de Lenguaje se observa también una mejora significativa en los niños (gráfico 1). $\mathrm{Al}$ respecto, las hermanas consagradas $\mathrm{y}$ misioneras desarrollaron diversas actividades con los niños ayudándoles a aprender palabras sencillas desde una edad muy temprana edad, motivándoles con dibujos, láminas ilustrativas y música. Un estudio sobre las repercusiones linguiísticas con 13 niños en situación de maltrato y nueve en abandono emocional, muestra que estos niños tienen un índice cognitivo general inferior a lo esperado y según módulos de morfología, que verificó el dominio del lenguaje en los niños, un $78 \%$ tenía un nivel de dominio morfológico y de sintaxis por debajo de su edad. ${ }^{17}$ Datos que se repiten en diferentes estudios, pero que no se encontraron en los niños de la Casa Hogar, posiblemente porque la mayoría ingresó en la Casa en condición de recién nacido o siendo lactante, viviendo ya desde sus primeros años de vida rodeado de un ambiente positivo y de constantes estímulos adecuados.

En el área social, por el contrario, no se observaron muchos cambios en los niños (gráfico 2). Garcia-Baamonte ${ }^{18}$ refiere que en los dos primeros años de infancia la relación con el cuidador principal es fundamental, después al ingresar al colegio y a la guardería, la socialización comienza a ser más estructurada, aunque la familia aún sigue siendo el punto de referencia y la calidad de la relación que los niños tendrán dependerá mucho de lo vivido en casa, siendo muy posible que los niños rechazados tengan relaciones más complicadas.

Respecto al área motora, los niños lograron mejora significativa (gráfico 3). Área importante en el desarrollo de habilidades motoras gruesas y finas para fortalecer el equilibrio corporal en relación con el espacio. No fue encontrado información de estudios previos al respecto, pero, a medida que avanza el desarrollo físico general del niño su capacidad de respuesta motora se amplía, tanto por la maduración física como por todas las oportunidades que se les otorga, como gatear, caminar, correr, saltar; además que sus movimientos se vuelven más complejos y ágiles. 
Los avances dependen mucho de los deseos de experimentar del niño, siendo que el juego se vuelve un vehículo de vital importancia para afrontar el mundo que los rodea, en relación con el espacio y los objetos, de saber y conocer quiénes son y tener un concepto de sí mismo, ${ }^{9}$ como necesidad primordial para la construcción de su personalidad. En ese sentido, el juego es un elemento eficaz para establecer una relación entre el cuerpo del niño y su entorno.

En el área de coordinación (gráfico 4), los datos no muestran mayores cambios. Esta área se desarrolla según las conductas motrices de base, como la postura y el equilibrio; siendo que es derivada del proceso de maduración de las estructuras neurológicas ${ }^{12}$ y mediante la actividad, como los movimientos de ejercicios coordinados entre sí. En la Casa Hogar los niños desarrollan múltiples actividades, además de imitarse entre sí, descubriendo en sus compañeros movimientos nuevos que los van incorporando.

Ante lo expuesto, la labor de la enfermera es importante en el equipo multiprofesional, al responsabilizarse del control del desarrollo

\section{Correspondencia:}

Marica Bussi

Correo electrónico: villasalvador@comunitacenaculo.it

\section{REFERENCIAS BIBLIOGRÁFICAS}

1. Moreno MJ. Estudio sobre las variables que intervinieron en el abandono físico o negligencia infantil comparativamente con otros tipos de maltrato infantil. [tesis doctorado]. España: Universidad de Extremadura, 2002.

2. Giménez-Pando J, Pérez-ArjonaE, Dujovny M, Díaz FG. Secuelas neurológicas del maltrato infantil. Revisión Bibliográfica. Neurocirugía [internet]. Abr 2007 [citado jul 14 de 2014]; 18 (2):95-100. Disponible en: http://scielo.isciii.es/pdf/neuro/v18n2/investig acion1.pdf

3. Ministerio de Salud Perú. Asociación Española de Padres con la custodia de los hijos [internet]. Lima (Perú): MINSA; 2009 [5 septiembre 2009; 29 noviembre 2011]. El 40\% de casos de maltrato infantil es ocasionado por madres [aproximadamente 01 lauda]. Disponible en: http//www.noticia- psicomotor como tarea preventiva y útil para detectar a tiempo deficiencias y alteraciones o trastornos en los niños que se podrían corregir con una intervención oportuna, especialmente porque puede trabajar en conjunto con los especialistas, como el terapista, psicólogo, nutricionista, entre otros, considerando la importancia del cuidado integral del menor, que incluye sus dimensiones físicas, emocionales y espirituales, reconociendo que el ser humano es un ser digno desde que nace.

Concluyendo, el estudio demuestra que los niños presentaron una buena evolución en su desarrollo psicomotor según grupo etario, por escala de evaluación (EEDP y TEPSI), aunque al analizarse los datos por áreas de desarrollo, los cambios fueron más concretos en el área de lenguaje y motora, datos que son relevantes principalmente porque se trata de niños que sufrieron abandono familiar y maltrato. Es necesario realizar otras investigaciones con niños menores de cuatro años, reconociendo la importancia de esta etapa en el desarrollo del ser humano. Debiendo ante todo, reconocer las limitaciones propias de un estudio exploratorio $\mathrm{y}$ descriptivo.

norte.com/2009/09/di/e-40-de-casos-demaltratoinfantil-co-ocasionado-por-la-madre

4. INEI. Instituto Nacional de Estadística e Informática. Disponible en: [www.gob.pe]. Perú.

5. Macarena L. Método María Montessori [internet]. 2003; oct. Santiago. Disponible en: www.elviajerosuizo.com/resources/método.m ontessori-resumen.pdf

6. Martínez OB, Céspedes CN. Metodología de la investigación. Estrategias para investigar. Ed.Libro Amigo. Lima Perú, 2008.

7. El Fondo de Naciones Unidas para la Infancia [internet]. Suiza: Asamblea General de los derechos del niño; 1990 [2 septiembre 1990; 28 noviembre 2011]. Convención sobre los derechos del niño [Aproximadamente 01 lauda]. Disponible en: 
www.unicef.org/Mexico/Spanish/mx_resource $\underline{\text { txtocdn }}$

8. Martínez A. Causas del maltrato infantil. Caracas-Venezuela: Ciencias de la Salud [internet]; $2002 \quad$ Disponible en:http://www.monografias.com/trabajos 10/m $\underline{\text { alin/malin.shtml }}$

9. Paul N. Tratado de Enfermería Nelson. $16^{\mathrm{a}}$ ed. España: Mc Graw Hill Interamericana; 2005.vol II, pp.118-120 Cap.35.

10. Verduz P, Alcaraz R. "Retraso Psicomotor". Developmental delay [internet] 2008 Dic. [citado may 13 de 2009]; 4(3): 2-8. Disponible en:

www.psiquiatria.com/buscador/proxy.ats...arti culos...- En

11. Ruiz CI. Impacto psicológico de la negligencia familiar (leve versus grave) en un grupo de niños y niñas. [tesis de doctorado]. España: Universidad de Málaga, 2002.

12. Centro de referencia latinoamericano para la educación preescolar [internet].Cuba: Dr. Franklin Martínez Mendoza; 2010 [citado jun 19 de 2010]. De Franklin Martínez M. La Estimulación Temprana: Enfoques, problemáticas y proyecciones. Disponible en: http://www.oei.org.co/celep/

13. Torres DB. Investigaciones sobre el desarrollo cerebral y emocional: sus indicativos en relación a la crianza.Rev.49 [internet]. 2010. Disponible en: www.sepypna.com/.../investigacionesdesarrollo-cerebral-emocional/pdf

14. Del corazón E. Comunidad Cenáculo. Destellos de Luz. Italia. Julio 2010

15. Gadea DL. Escuelas para padres y maestros. Confianza contra desconfianza. México; 2012. Disponible en: Presencias.net/educar/ht1024.htlm

16. Antoinette A. Teoría del apego J.Bolwbye. M. Aisworth. [internet] [citado nov 01 de 2013]

17. Manso M. Estudio sobre las repercusiones lingüísticas del maltrato y abandono emocional infantil. Rev. Logopedia, Foniatra y Audiología. [internet].2003 [p211-222]
18. Baamonde SE. Análisis de la competencia lingüística y de la adaptación personal, social, escolar y familiar en niños institucionalizados en centros de acogida [tesis doctoral]. España: Universidad de Extremadura, 2008. 